\title{
Enquête ethnobotanique sur les Euphorbiaceae médicinales utilisées chez les Baoulé du District de Yamoussoukro (Côte d'Ivoire)
}

\author{
SARAKA Allou Isidore ${ }^{1 *}$, CAMARA Djeneb ${ }^{1}$, BENE Kouadio'12 et ZIRIHI Guédé Noël1 \\ ${ }^{1 *}$ Laboratoire de Botanique, UFR Biosciences, Université Félix Houphouët Boigny de Cocody Abidjan ; 22 BP 582 \\ Abidjan 22 \\ 2Laboratoire de Botanique et Phytothérapie, UFR des Sciences de la Nature, Université Nangui Abrogoua, Abidjan ; \\ 02 bp 801 Abidjan 02 \\ Auteur correspondant, email: asaraka01@yahoo.fr / cel : (+225) 07790483
}

Original submitted in on $26^{\text {th }}$ March 2018. Published online at www.m.elewa.org on $30^{\text {th }}$ June 2018 https://dx.doi.org/10.4314/jab.v126i1.11

\begin{abstract}
RESUME
Objectif: Une enquête ethnobotanique a été menée dans la District de Yamoussoukro dans le but de connaitre les Euphorbiaceae utilisées traditionnellement par les Baoulé pour leurs soins de santé..

Méthodologie et résultats: Une étude ethnobotanique a été réalisée auprès de quarante (40) tradipraticiens de santé du District. Douze (12) espèces réparties en huit (8) genres ont été répertoriées. Les feuilles (y compris rameaux feuillés) constituent les organes les plus utilisés (59\%). La décoction (34\%) est le mode de préparation le plus fréquemment employé pour la majorité des recettes médicamenteuses. La voie orale est la plus sollicitée avec $44 \%$ des recettes. Par ailleurs, l'étude a révélé que les maladies de la peau (34\%) ainsi que les affections du tube digestif (29\%) sont les plus traitées avec les Euphorbiaceae suivi par le traitement des troubles respiratoires (14\%) et celui des affections ostéo-articulaires (11\%).

Conclusion et application des résultats: Ces résultats peuvent être considérés comme une source d'information pour les recherches scientifiques dans le domaine de la phytochimie et de la pharmacologie.

Mots clés : Ethnobotanique, Euphorbiaceae, Baoulé, District de Yamoussoukro.
\end{abstract}

\section{ABSTRACT:}

Objective: An ethnobotanical study was conducted in the District of Yamoussoukro in order to know the Euphobiaceae traditionally used by the Baoulé for health care

Methodology and results: Ethnobotanical surveys were carried out from forty (40) traditional healers of the District. Twelve (12) species of medicinal plants divided into eight (08) genera were listed. Leafs (including small branches of leafs) constitute the most used organs (59\%). The decoction (34\%) is the mode of preparation the most frequently employed for the majority of the medicamentous formula. The oral way is requested with $44 \%$ of the formula. In addition, the study revealed that the skin diseases (34) as well as the affections of the digestive tract $(29 \%)$ are treated with Euphorbiaceae followed by the treatment of the respiratory disorders (14\%) and that of the ostéo-articular affections $(11 \%)$.

Conclusion and application of results: These results can be considered as source of information for phytochemical and pharmacological researches..

Key words: Ethnobotanique, Euphorbiaceae, Baoule, District of Yamoussoukro 


\section{INTRODUCTION}

L'ethnobotanique est l'étude de l'interaction socioculturelle entre les plantes et les peuples de manière empirique. Selon I'Organisation Mondiale de la Santé (OMS), environ 65 à $80 \%$ de la population mondiale dans les pays en développement dépend essentiellement des plantes pour leurs soins de santé primaires en raison de la pauvreté et du manque d'accès à la médecine moderne (Awoyemi et al., 2012). La Côte d'ivoire n'échappe pas à cette assertion, la médecine traditionnelle y occupe une place prépondérante pour les soins de santé primaires pour une grande majorité de personnes. De nombreuses investigations ethnobotaniques ont été menées dans la plupart des pays en développement en Afrique (NacoulmaOuedraogo et al., 2007) et particulièrement en Côte d'Ivoire (Tra Bi et al., 2008). Ces enquêtes ethnobotaniques qui ont été menées, visaient le plus souvent une affection bien définie ou consistaient en un inventaire de toutes les plantes utilisées par la population cible. II en ressort que les plantes recensées appartiennent à plusieurs familles botaniques. Cependant les études qui visent les espèces végétales d'une famille

\section{MATERIEL ET METHODES D'ETUDE}

Matériel végétal: II est constitué de l'ensemble des espèces végétales médicinales de la famille des Euphorbiaceae (herbes, lianes, arbustes et arbres) répertoriées et récoltées dans le District de Yamoussoukro.

Méthodes d'étude: La méthode d'échantillonnage stratifié probabiliste (Tra Bi et al., 2008) a été utilisée. Elle consiste à diviser la zone d'étude en différentes strates et à y associer le même nombre d'enquêtés. Nous avons réalisé un catalogue des Euphorbiaceae médicinales de Côte d'Ivoire à partir des différents travaux et ouvrages consultés à savoir: Adjanohoun et al., (1979), Aké-Assi (1984, 2011), Zirihi (1991), N'Guessan (2008), N'guessan et al., (2010), Béné et al., (2016).

Sites de collecte des plantes: Le District de Yamoussoukro est composé de quatres (4) Souspréfectures que sont Attiégouakro, Lolobo, Yamoussoukro et Kossou. Dans ce travail, chaque sous-préfecture a constitué une strate. Dans chacune botanique spécifique restent très peu disponibles. Les études effectuées en Côte d'ivoire ont révélé une richesse de la flore dans laquelle la famille des Euphorbiaceae a attiré notre attention. Cette famille a été majoritairement citée lors des études réalisées dans plusieurs régions de la Côte d'Ivoire, à savoir : Zirihi (1991) chez les Bété d'Issia 4,76\%, Ouattara (2006) à Divo 8,8\%, N'guessan (2008) 6,27\% chez les peuples Abbey et Krobou d'Agboville, N'guessan et al., (2010) $15,15 \%$ dans la région d'Agboville et Béné et al., (2016) dans le Département de Transua $8,51 \%$. C'est ce qui justifie la présente enquête ethnobotanique sur les plantes médicinales appartenant à la famille des Euphorbiaceae dans le District de Yamoussoukro. Cette étude consiste à collecter des informations concernant les usages thérapeutiques des plantes de cette famille à partir d'un catalogue que nous avons élaboré et présenté aux tradithérapeutes de cette circonscription. II s'agira spécifiquement d'identifier les plantes de la Famille des Euphorbiaceae utilisées par les populations du District de Yamoussoukro pour leurs soins de santé.

des strates, cinq (05) sites ont été visités, et deux (2) tradipraticiens de santé y ont été consultés. Ce sont :

Sous-préfecture de Lolobo (Strate 1) : Tounzuebo, Menou, Ouffoue-Diekro, Assanou et Koriakro.

Sous-préfecture d'Attiégouakro (Strate 2) : Attiegouakro, Abokouamekro, Aka-yaokro, KacouBroukro, Mahounou-Nanafoue.

Sous-préfecture de Yamoussoukro (Strate 3) :

Seman, Logbakro, Nanan, Bonzi et Akpéssékro

Sous-préfecture de Kossou (Strate 4) : Zatta, Toumbokro, Kossou, Gogokro et Bocabo.

Tradipraticiens de santé rencontrés

Les enquêtes ethnobotaniques basées sur la méthode d'entretien semi-structuré (Klotoé et al., 2013) ont été menées auprès de quarante (40) guérisseurs selon leur disponibilité. Les critères de choix des guérisseurs ont résidé sur leur audience ainsi que la reconnaissance des images ou échantillons que nous leur avions apportés. Nous avons effectué deux à trois visites 
auprès de tous. Pour la plupart c'est d'abord une visite de courtoisie, puis une ou deux autres pour faire l'enquête proprement dite. La fiche d'enquête comportait des questions sur le nom local de l'espèce, les organes ou parties de la plante utilisée, les modes de préparation et d'administration des recettes, l'état d'utilisation (frais ou sec), les maladies traitées etc. Les questions ont été posées en Baoulé, la langue locale comprise de tous. Nous nous sommes faits accompagner d'un guide interprète bien connu de la Région pour avoir servi à l'Agence Nationale d'Appui au Développement Rural. Les entretiens ont été réalisés de deux manières. Avec certains tradithérapeutes, nous leurs avons présenté le catalogue de plantes médicinales de la famille des Euphorbiaceae de Côte d'Ivoire mentionné plus haut, puis ensemble nous avons identifié et récolté des plantes au cours d'une sortie en brousse comme le suggère Cunningham (2002). Avec d'autres nous avons, à partir de notre catalogue, récolté des échantillons en vue de les faire identifier et valider. Dans les deux cas nous leur avons posé plusieurs questions partant des noms vernaculaires aux usages thérapeutiques de celles-ci.

Collecte des plantes et confection des herbiers: Des échantillons frais de plantes ont été récoltés. Une planche d'herbier a été confectionnée depuis le lieu de récolte des plantes pour chacune d'elles. Après la récolte des échantillons et la confection des herbiers, la confirmation de l'identité des espèces végétales a été faite sur le terrain, pour l'ensemble des espèces. La nomenclature retenue pour ces plantes recensées suit la version de la classification phylogénétique, selon les travaux de l'Angiosperms Phylogeny Group, dite APG III (2009).

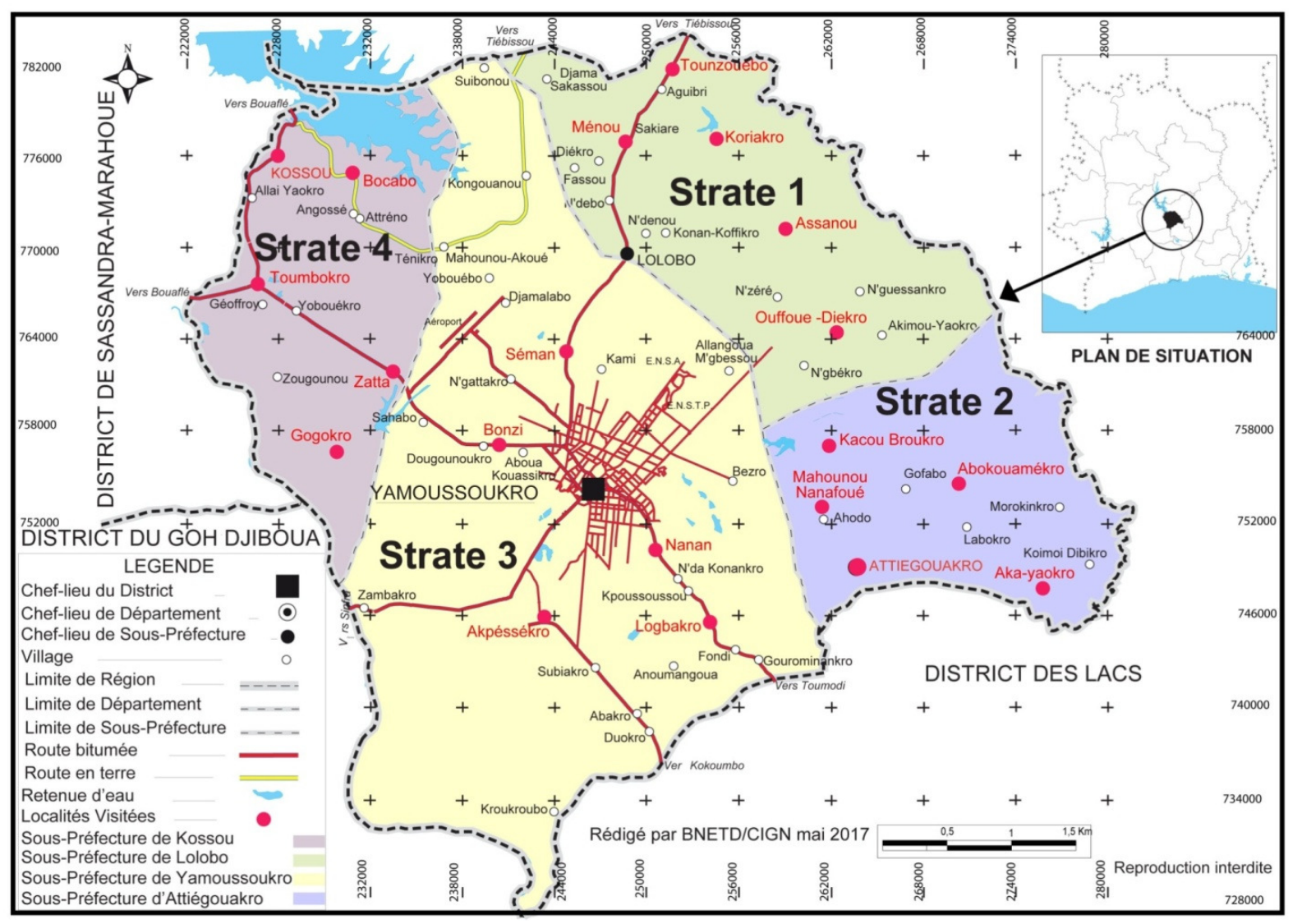

Figure 1 : District de Yamoussoukro

\section{RESULTATS ET DISCUSSION}

Tradipraticiens de santé rencontrés : Quarante (40) tradipraticiens de santé du District de Yamoussoukro ont été enquêtés. II y a parmi eux 24 hommes et 16 femmes (Tableau I). Les résultats montrent que cet art médical est majoritairement pratiqué par les hommes qui représentent $60 \%$ contre $40 \%$ de femmes. Ces résultats sont similaires à ceux obtenus par Gnagne et al., (2017) dans le Département de Zuénoula et qui 
montrent que les hommes sont les plus représentés avec $65 \%$ contre $35 \%$ de femmes. Ils sont contraires à ceux obtenus en Côte d'Ivoire par Béné et al. (2016) qui montraient $66,67 \%$ de femmes. Cette différence est certainement due au fait que nous avons consulté des guérisseurs et non des herboristes dans cette enquête. Investigation botanique : Les investigations réalisées dans le District de Yamoussoukro nous ont permis d'identifier douze (12) espèces réparties en huit (08) genres de la famille des Euphorbiaceae. Les plantes recensées et leurs indications thérapeutiques sont regroupées dans le Tableau II. Dans cette étude, le genre Euphorbia (avec 3 espèces) est le plus rencontré soit $66,67 \%$ du total. Cela pourrait s'expliquer par le fait que la zone d'étude soit favorable à la croissance de ce genre. Ce résultat est similaire à celui observée par Aké-Assi (1984) au cours de l'étude descriptive et biogéographique réalisée sur la flore de la Côte d'ivoire. Dans cette étude le genre Euphorbia a été majoritairement cité dans la famille des Euphorbiaceae.

\section{Biologie, morphologie et biogéographie}

Du point de vue des types biologiques (Figure 2), les phanérophytes avec 09 espèces sont les plus abondants avec $75 \%$ et repartis comme suit : les microphanérophytes (mp) avec 04 espèces soit $33,33 \%$, les mésophanérophytes (mP) avec 1 espèce soit $8,33 \%$ et les nanophanérophytes (np) avec 04 espèces soit $33,33 \%$. Les microphanérophytes et les nanophanérophytes avec $33,33 \%$ chacun de l'ensemble des types biologiques sont donc les plus représentés. Ce résultat est similaire à celui d'autres auteurs qui ont montré à des proportions différentes que les microphanérophytes sont majoritairement sollicités dans les différentes études qu'ils ont menées: Ouattara (2006) avec 42\%, N'guessan (2008) avec $35,18 \%$, Lakouéténé et al. (2009) avec $40 \%$ et Béné et al. (2016) avec 36,17\%. Quant aux types morphologiques, les plantes recensées sont composées d'une (1) espèce d'arbre, six (06) espèces d'arbustes et arbustes sarmenteux et cinq (05) espèces herbacées. Les arbustes avec 50,00\% des espèces sont majoritaires (Figure 3). Cela pourrait s'expliquer par le fait que Yamoussoukro est situé dans une zone de transition entre la forêt et la savane. Notre résultat est proche de celui de Béné et al. (2016) qui révèle que les arbustes avec $51,09 \%$ sont les plus représentés dans les résultats de ses enquêtes. Contrairement à ces résultats d'autres auteurs comme, N'Guessan et al. (2010) et Diatta et al. (2013) ont trouvé que les arbres étaient majoritaires, quand Zirihi (1991) et N'Guessan (2008) trouvaient que les herbes étaient les plus sollicitées.

Tableau1 : Informations sur les tradipraticiens de santé enquêtés

\begin{tabular}{|l|c|c|c|c|c|c|c|c|c|}
\hline STRATES & \multicolumn{2}{|c|}{ STRATE 1 } & \multicolumn{2}{c|}{ STRATE 2 } & \multicolumn{2}{c|}{ STRATE 3 } & \multicolumn{2}{c|}{ STRATE 4 } & \\
\hline SEXE & Hommes & Femmes & Hommes & Femmes & Hommes & Femmes & Hommes & Femmes & TOTAL \\
\hline EFFECTIF/SEXE & 7 & 3 & 2 & 8 & 6 & 4 & 9 & 1 & 40 \\
\hline EFFECTIF /STRATE & \multicolumn{2}{|c|}{10} & \multicolumn{2}{c|}{10} & \multicolumn{2}{c|}{10} & & 10 & 40 \\
\hline
\end{tabular}


Saraka et al, J. Appl. Biosci. 2018 Enquête ethnobotanique sur les Euphorbiaceae médicinales utilisées chez les Baoulé du District de Yamoussoukro (Côte d'Ivoire)

Tableau 2 : Liste des Euphorbiaceae médicinales utilisées chez les Baoulé du District de Yamoussoukro (Côte d'Ivoire)

\begin{tabular}{|c|c|c|c|c|c|c|c|c|c|}
\hline $\mathrm{N}^{\circ}$ & $\begin{array}{l}\text { Noms } \\
\text { scientifiques }\end{array}$ & $\begin{array}{l}\text { Noms } \\
\text { vernaculaires }\end{array}$ & $\begin{array}{l}\text { Type } \\
\text { Mph }\end{array}$ & $\begin{array}{l}\text { Type } \\
\text { Biologique }\end{array}$ & $\begin{array}{l}\text { Type } \\
\text { Biogéographie }\end{array}$ & $\begin{array}{l}\text { Parties } \\
\text { utilisées }\end{array}$ & $\begin{array}{l}\text { Indication } \\
\text { thérapeutique }\end{array}$ & $\begin{array}{l}\text { Mode de } \\
\text { préparation }\end{array}$ & Mode d'administration \\
\hline 1 & $\begin{array}{l}\text { Alchornea cordifolia } \\
\text { (Schum. \&Thonn.) } \\
\text { Müll.Arg. }\end{array}$ & Djéka & Arb & $\operatorname{Lmp}$ & GC-SZ & Feuilles & $\begin{array}{l}\text { Mycoses cutanées } \\
\text { Lombalgie Ulcère }\end{array}$ & Décoction & $\begin{array}{l}\text { Se laver le décocté jusqu'à } \\
\text { la guérison Boisson }\end{array}$ \\
\hline 2 & Croton hirtus L'Hérit. & Ahoublé-aliè & $h$ & $\mathrm{np}$ & GC & Plante entière & Constipation & Pétrissage & Lavement \\
\hline 3 & Croton lobatus Linn. & N'gnienbô-laliè & $h$ & Th & GC-SZ & Plante entière & Hypertension & Décoction & Boire le décocté \\
\hline 4 & $\begin{array}{l}\text { Elaeophorbia grandifolia } \\
\text { (Haw) }\end{array}$ & Lôlô & Arb & $\mathrm{mp}$ & GC-SZ & Feuilles & Hoquet, Asthme & $\begin{array}{l}\text { Ramollissem } \\
\text { ent et } \\
\text { expression }\end{array}$ & Instillation buccale \\
\hline 5 & $\begin{array}{l}\text { Euphorbia heterophylla } \\
\text { Linn. }\end{array}$ & Gbamlo-aliè & $h$ & Th & GC & Feuilles & \begin{tabular}{|l} 
Indigestion, \\
Dysenterie \\
\end{tabular} & Décoction & Boisson du décocté \\
\hline 6 & Euphorbia hirta Linn. & Ako-Lôlô & $h$ & $\mathrm{Ch}$ & GC-SZ & $\begin{array}{l}\text { Plante } \\
\text { entière } \\
\text { Inflorescence }\end{array}$ & $\begin{array}{l}\text { Mycoses cutané, } \\
\text { diarrhée. -Maux de } \\
\text { ventre }\end{array}$ & \begin{tabular}{|l} 
Décoction, \\
Infusion \\
Mastication \\
avec grains \\
d'arachide
\end{tabular} & $\begin{array}{l}\text { Boisson de l'infusé, bain du } \\
\text { décocté , Avaler le jus de } \\
\text { la mastication }\end{array}$ \\
\hline 7 & Euphorbia hypericifolia & $\begin{array}{l}\text { Ako Lôlô } \\
\text { N'vasuè }\end{array}$ & $\mathrm{H}$ & $\mathrm{np}$ & GC-SZ & Feuilles & $\begin{array}{l}\text { Diarrhée, Dysenterie, } \\
\text { Colique }\end{array}$ & Pétrissage & Lavement \\
\hline 8 & Jatropha curcas Linn. & Aplohploh & Arb & $\mathrm{np}$ & GC-SZ & Feuilles & \begin{tabular}{|l|} 
Diarrhée, \\
Saignement
\end{tabular} & \begin{tabular}{|l|} 
Décoction \\
Expression \\
\end{tabular} & $\begin{array}{l}\text { Boisson du décocté, } \\
\text { Instillation nasale }\end{array}$ \\
\hline 9 & Jatropha gossypiifolia & $\begin{array}{l}\text { Aplohploh } \\
\text { wokluè }\end{array}$ & Arb & $\mathrm{np}$ & GC-SZ & Feuilles & Hoquet, Asthme & Teinture & $\begin{array}{l}\text { Boire la teinture et masser } \\
\text { le thorax avec le résidu }\end{array}$ \\
\hline 10 & $\begin{array}{l}\text { Manihot esculenta } \\
\text { Crantz }\end{array}$ & Agba & Arb & $\mathrm{mp}$ & I & Feuilles & Ménorragie & $\begin{array}{l}\text { Expression + } \\
\text { Kaolin }\end{array}$ & Boisson \\
\hline 11 & $\begin{array}{l}\text { Ricinodendron } \\
\text { heudelotii (Baill.) Pierre } \\
\text { ex. Pax. }\end{array}$ & Apki & $A$ & $\mathrm{mP}$ & GC & $\begin{array}{l}\text { Ecorces de } \\
\text { tiges }\end{array}$ & $\begin{array}{l}\text { Ulcère gastrique, } \\
\text { œdèmes, plaies }\end{array}$ & Décoction & $\begin{array}{l}\text { Boisson, laver la plaie avec } \\
\text { le décocté jusqu'à la } \\
\text { guérison, lavement. }\end{array}$ \\
\hline 12 & Ricinus communis Linn. & Atténdé & Arb & $\mathrm{mp}$ & I & $\begin{array}{l}\text { Graines } \\
\text { Feuilles }\end{array}$ & $\begin{array}{l}\text { Teigne, dartre, } \\
\text { boutons, panaris, } \\
\text { Zona, Rhumatisme, } \\
\text { Entorse }\end{array}$ & $\begin{array}{l}\text { Calcination, } \\
\text { pâte + huile, } \\
\text { Ramollissem } \\
\text { ent au feu }\end{array}$ & $\begin{array}{l}\text { Frotter sur le corps ou la } \\
\text { partie infectée }\end{array}$ \\
\hline
\end{tabular}

Légende:

mP: mésophanérophyte ; mp: microphanérophytes; np: nanophanérophyte; Th: Thérophyte; Ch: Chamephyte; A: Arbre; Arb: Arbuste; h: Herbe

GC: Espèce Guinéo-Congolaise ; GC-SZ: Espèce Guinéo-Congolaise et Soudano-Zambézienne; i: Espèce introduite 


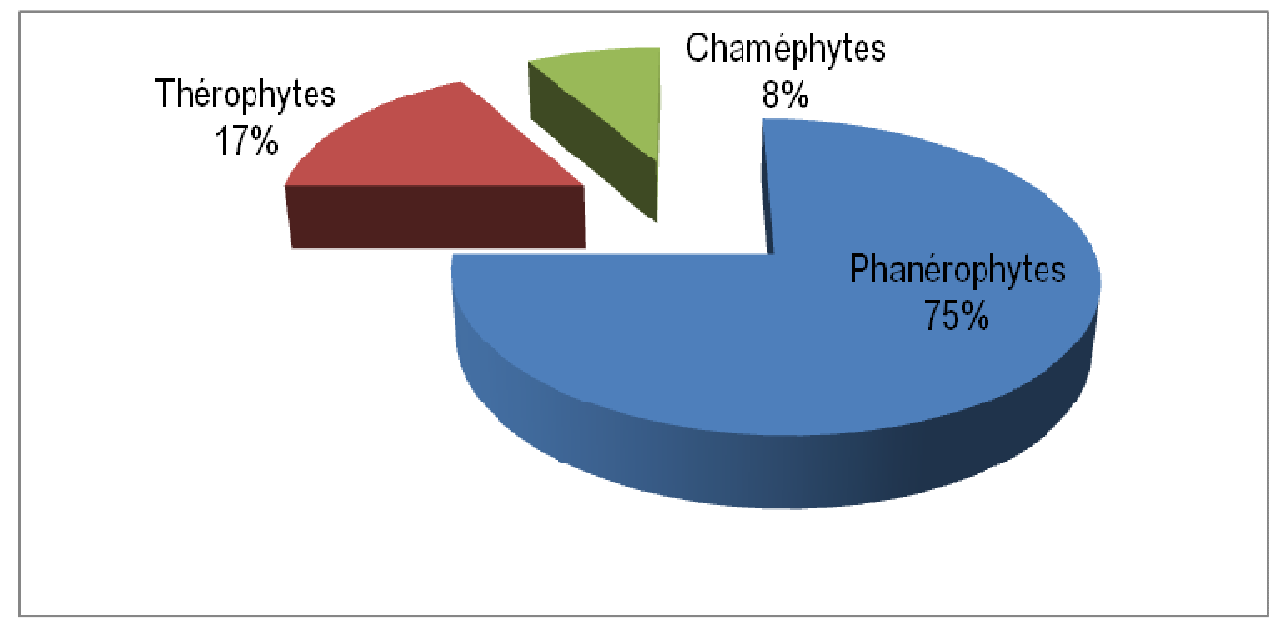

Figure 2: Spectre des différents types biologiques

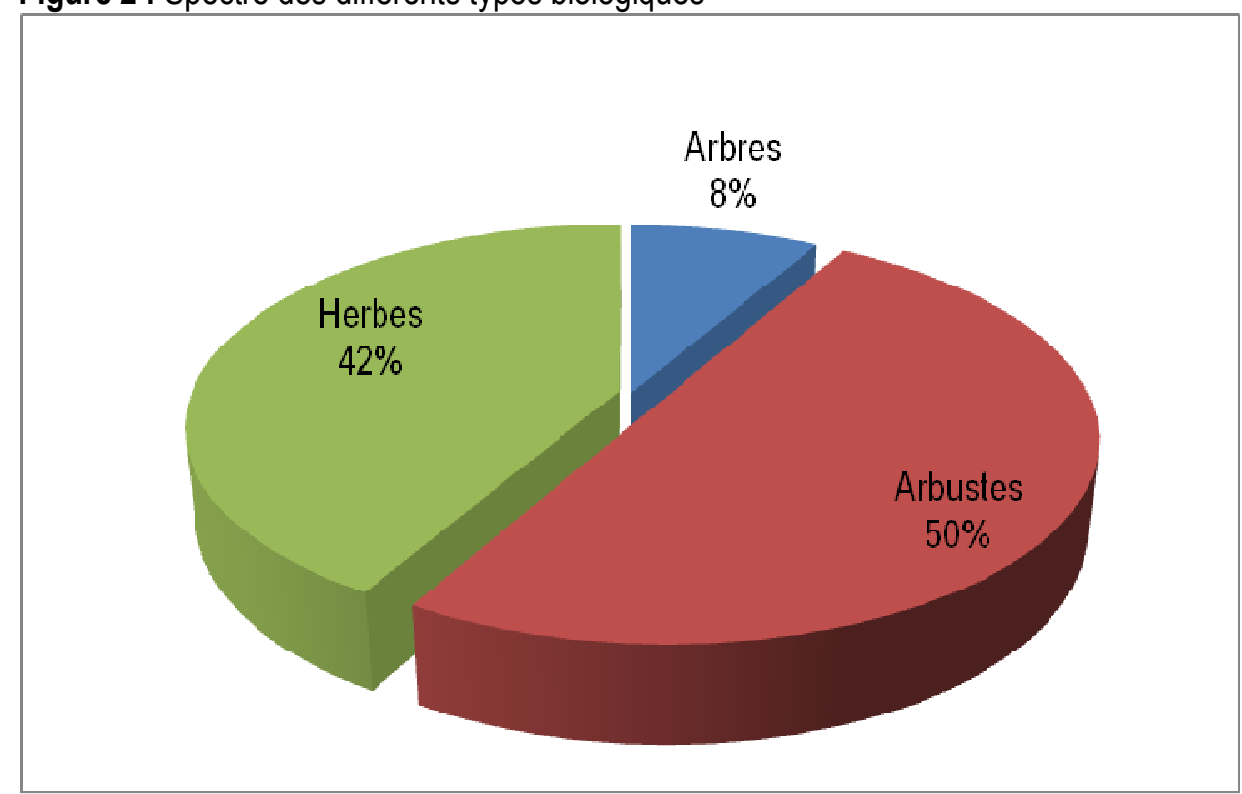

Figure 3: Spectre des différents types morphologiques

Au niveau des types phytogéographiques (Figure 4) les espèces communes aux régions phytogéographiques Guinéo-Congolaise et Soudano-Zambézienne (GC-SZ) avec 07 espèces soit $58,33 \%$ sont les plus représentées. Elles sont suivies des espèces des régions phytogéographiques Guinéo-Congolaise (GC) avec 3 espèces soit $25,00 \%$. Les espèces introduites (i) avec 02 espèces représentent $16,67 \%$ des espèces recensées. La situation géographique du District de Yamoussoukro qui est une zone de transition entre la forêt et la savane pourrait justifier le fort taux des espèces communes aux régions Guinéo-Congolaise et Soudano-Zambézienne (GC-SZ).

Partie des plantes utilisées: Les feuilles (y compris les rameaux feuillés) sont les parties les plus utilisées avec 59\% (Figure 5). Ces résultats sont similaires à ceux obtenus par plusieurs auteurs qui ont montré à travers leurs études ethnobotaniques que les feuilles sont les parties majoritairement utilisées dans les différentes préparations thérapeutiques : Zirihi (1991) avec 64,49\%, Tra Bi (1997) avec 70\%, Ouattara (2006) avec 44,26\%, Ambé et al. (2015) avec $28 \%$,Yapi et al. (2015) avec $43,18 \%$, Béné et al. (2016) avec 63,96\%. Viennent ensuite la plante entière avec $25 \%$, suivie des écorces de tige et les graines qui représentent chacune $8 \%$ des parties utilisées.

Modes de préparation des recettes: Différentes techniques sont utilisées pour la préparation des recettes dont la plus fréquente est la décoction (42\%). $\mathrm{Ce}$ résultat est similaire à celui des auteurs : 
Adjanohoun et Aké Assi (1979) avec 32,94\%, Ambé et al. (2015) avec 79\%, Béné et al. (2016) avec 36\%. Ceci peut se justifier par le fait que la décoction permet de recueillir le plus de principe actifs, par ailleurs la décoction permet d'atténuer ou annuler la toxicité de certaines plantes selon la plupart des tradipraticiens rencontrés. L'expression (25\%) et le pétrissage $(25 \%)$ sont les modes fréquemment utilisés après la décoction (Figure 6).

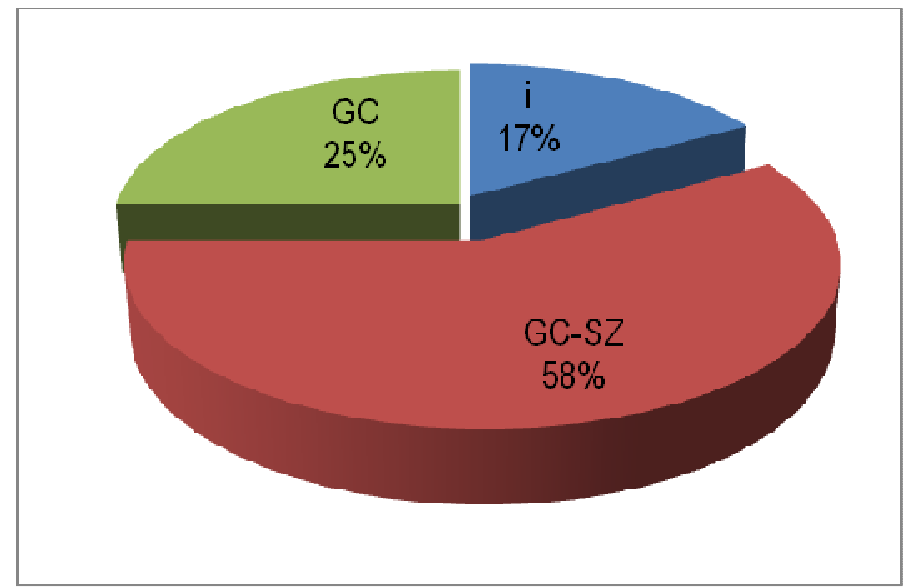

\section{Légende}

GC: Espèce Guinéo-Congolaise

GC-SZ: Espèce Guinéo-Congolaise et

Soudano-Zambézienne

i: Espèce introduite

Figure 4: Spectre des différents types phytogéographiques

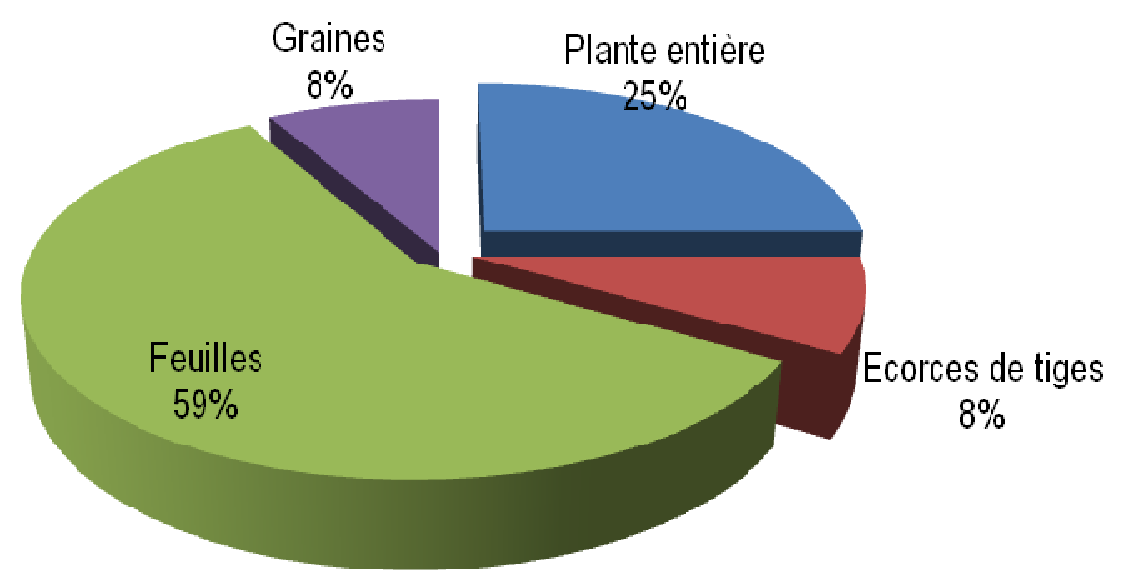

Figure 5 : Spectre des différentes parties de plantes utilisées 


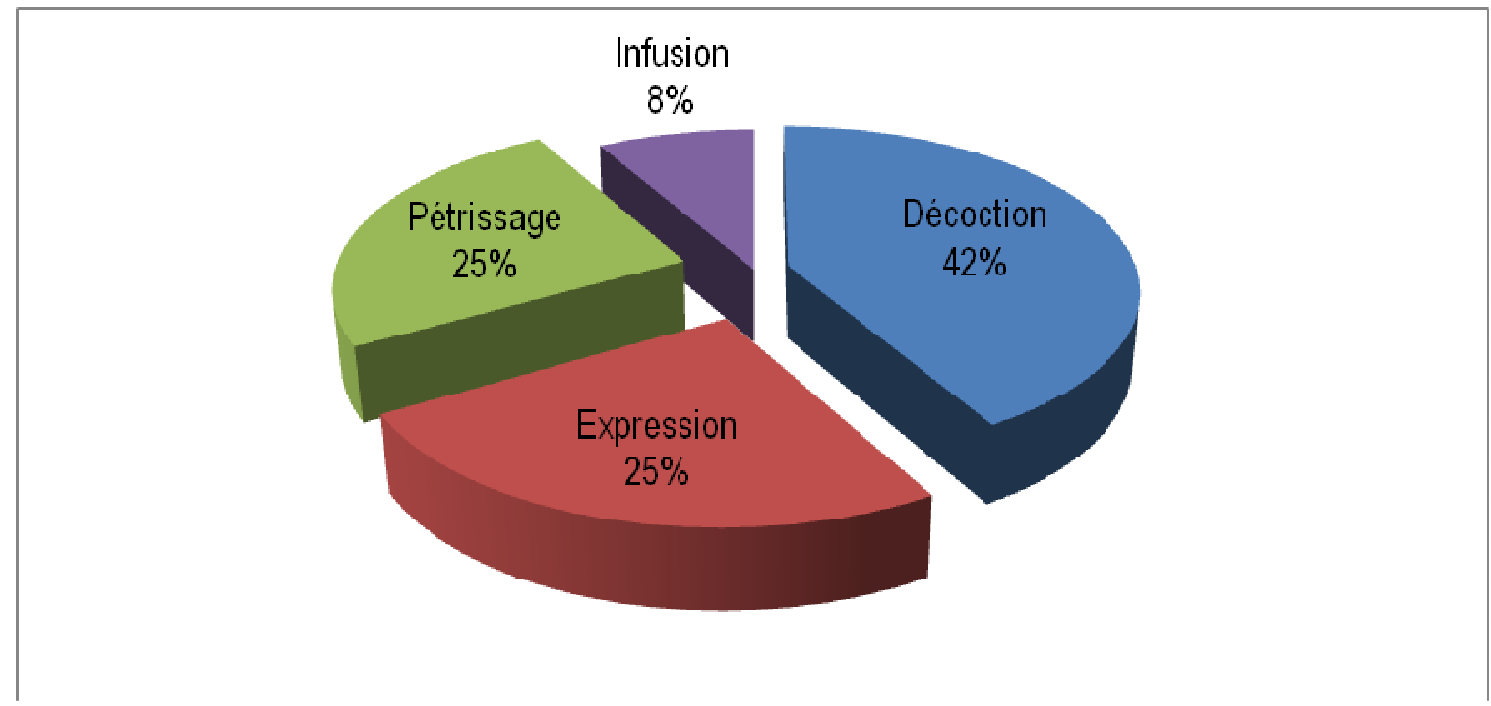

Figure 6 : Spectre des différentes techniques de préparation

Voies d'administration des préparations: Différentes voies d'administration ont été enregistrées parmi lesquelles, la voie orale (44\%) est la plus sollicitée (Figure 7). Ce résultat est conforme à celui de N'Guessan (2008) qui dans ses études a montré que le breuvage est la voie d'administration la plus utilisée. En effet, dans le traitement des infections bactériennes, fongiques et/ou parasitaires localisées dans des organes profonds, le transite des médicaments par l'appareil digestif facilite leur assimilation et leur action (Tra Bi et al. , 2008).

Indications thérapeutiques: Les 12 Euphorbiaceae identifiées sont cités dans le traitement de 14 affections (Figure 8), symptômes, phénomènes morbides que nous avons regroupé en 7 ensembles de maladies (affections dermatologiques, affections respiratoires, affections cardio-vasculaires, affections uro-génitales, affections métaboliques, affections du tube digestif et les affections ostéo-articulaires). De ce regroupement (Figure 9), il ressort que les affections dermatologiques (34\%) ainsi que les affections du tube digestif (29\%) sont les plus traitées avec les Euphorbiaceae dans le District de Yamoussoukro suivi par le traitement des troubles respiratoires $(14 \%)$ et les affections ostéoarticulaires (11\%). Ces valeurs traduisent d'une part le profil sanitaire de la Côte d'Ivoire fortement dominée par les maladies infectieuses et parasitaires (PNLP, 2005). D'autre part, elles traduisent l'importance des Euphorbiaceae dans le traitement des maladies dermatologiques qui pourrait être due à la composition de celles-ci en métabolites secondaires.
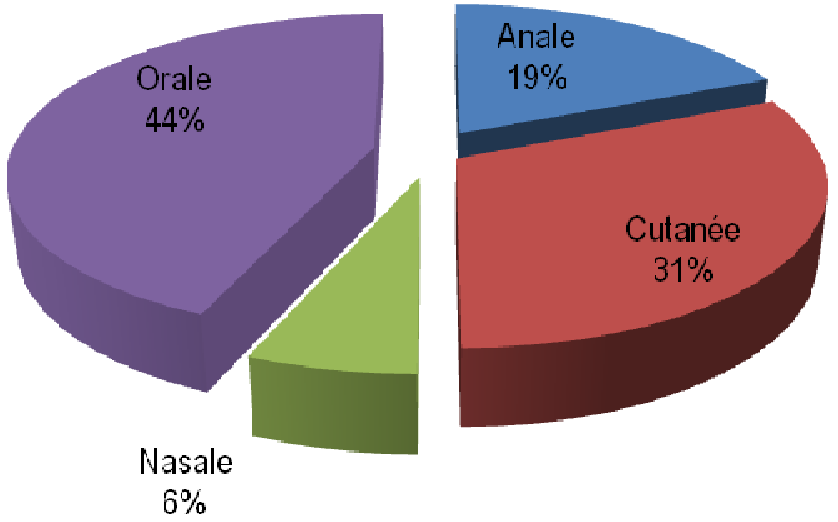
Figure 7: Principales voies d'administration des recettes

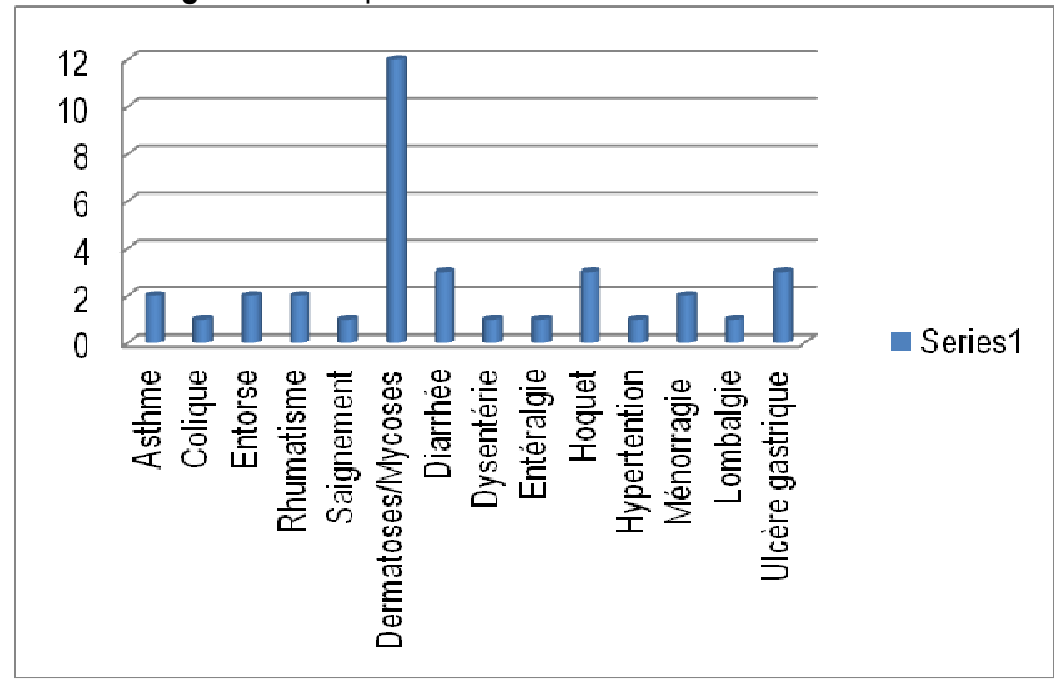

Figure 8: Principales indications thérapeutiques

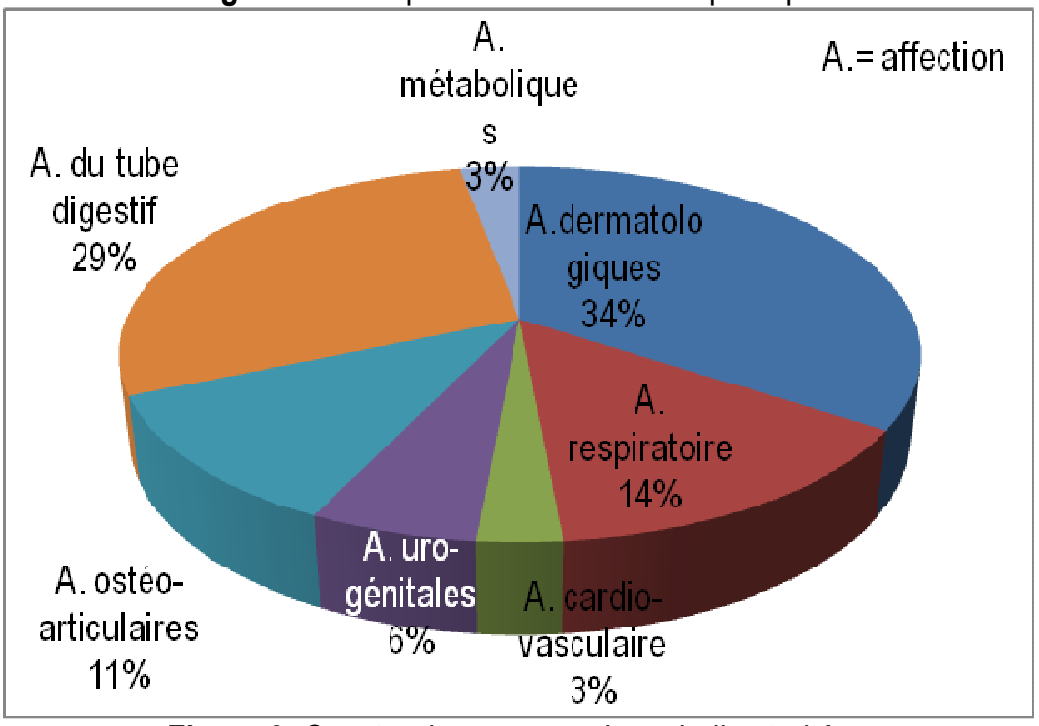

Figure 9: Spectre des groupes de maladies traitées

Association avec d'autres plantes et/ou ingrédients : Le traitement des différentes pathologies est prédominé par les recettes monospécifiques. Cette observation est confirmée par Monnet (2013) qui affirme que $92,59 \%$ des recettes sont mono spécifiques. Les quelques rares associations sont faites avec des excipients. Ceci pourrait traduire la maîtrise des plantes et des pathologies par les tradithérapeutes rencontrés au vue de la précision avec laquelle les plantes sont utilisées pour chaque pathologie. Ceci a l'avantage de prévenir les interactions médicamenteuses dangereuses pour les patients. En effet, il a été constaté que 30\% environ des interactions médicamenteuses mortelles en Afrique sont dues à l'usage des mixtures (El-Said et al. , 1969).

Etudes antérieures effectuées sur quelques Euphorbiaceae médicinales recensées: Nous présentons dans cette partie, l'activité pharmacologique et la composition phytochimique qui justifie l'usage traditionnelle de quelques plantes médicinales recensées:

Alchornea cordifolia (Shum.et Thonn.) Müll. Arg (Euphorbiaceae) : Les feuilles renferment des tannins, des alcaloïdes, des anthraquinones et des saponines capables d'inhiber la croissance d'helicobacter pyloris, de salmonella typhi, d'Echerichia coli et de shigella. Les extraits de feuilles ont montré un grand potentiel pour 
traiter l'ulcère gastrique et la diarrhée provoquée par les bactéries citées précedemment (Adeleye et al. , 2008). Les extraits méthanoliques de feuilles, de racines et d'écorces possèdent des propriétés antibactériennes. L'analyse phytochimique de ces extraits a montré la présence de polyphénols, de tanins, de triterpènes et de stérols (Mambé et al. , 2016). Mavar-Manga et al. (2008) ont isolé par fractionnement des extraits de racines et de feuilles d'Alchornea cordifolia, six composantes qui présentent un effet anti-inflammatoire significatif sur les œdèmes de souris. Certains auteurs ont montrés que des extraits acétate d'éthyle aussi bien que le macérat aqueux de feuilles d'Alchornea cordifolia auraient des effets inhibiteurs dose-dépendants sur le péroxyde d'hydrogène produit par les polynucléaires neutrophiles humains stimulés (Kouakou-Siransy et al., 2012). Owhe-Ureghe et al. (2016) ont montré par des tests in vitro que Alchornea cordifolia présente des activités antibactériennes sur Kocuria varians, Streptococcus pyogenes, Klebsiella pneumoniae, Enterobacter aerogenes et Salmonella enterica.

Euphorbia hirta : Les recherches bibliographiques ont révélé qu'Euphorbia hirta contient les composé

\section{CONCLUSION}

Cette étude a permis de recenser 12 espèces réparties en 8 genres. Quarante tradipraticiens de santé du District ont été interrogés dont 24 hommes et 16 femmes. Les phanérophytes sont les plus abondants $(75 \%)$. Les feuilles constituent les organes les plus utilisés $(59 \%)$ et la décoction (34\%) est le mode de préparation le plus employé. La voie orale est la plus sollicitée avec $44 \%$ des recettes. Les maladies de la suivants: afzeline, quercitrine, myricitrine, rutine, acide gallique, Euphorbine-A et Euphorbine-B, Euphorbine-C, Euphorbine-D, ß-amyrine, 24-methylene cyclo artenol, ß-sitosterol, heptacosane, n-nonacosane, l'acide shikmic, tinyatoxin, choline, camphol et des dérivées quercitol contenant rhamnose et l'acide chtolphenolic (Rastogi et Mehrotra, 2002). Cette plante possède des propriétés antibactériennes, anti-inflammatoires, antipaludiques, galactogeniques, antiasthmatiques, antidiarrhées, anticancéreuses, antioxydantes, antifertilités, antiamibiennes et activités antimycosiques (Sood et al. , 2005).

Ricinodendron heudelotii: Les amandes de Ricinodendron heudelotii sont composées de : matière sèche $92 \%$; protéines $24 \%$; matières grasses $48,7 \%$; sucres $0,084 \%$; amidon $0,42 \%$; cellulose $2,4 \%$. Les taux d'éléments minéraux sont : $0,33 \%$ de calcium ; $0,2 \%$; magnésium $0,033 \%$ de chlore ; $1,7 \%$ de phosphore et $0,8 \%$ de potassium (Saki et al. ,2005). Elles sont relativement riches en protéines, en matières grasses, en phosphore et en potassium.

peau (34\%) ainsi que les affections du tube digestif (29\%) sont les plus traitées. Ces données peuvent être considérées comme une source d'information pour les recherches dans le domaine de la phytochimie et de la pharmacologie en vue de déterminer les bases scientifiques qui sous-tendent l'utilisation de ces plantes.

\section{REMERCIEMENTS}

Nos remerciements vont à l'endroit du Centre National de Floristique (CNF) de I'Université Félix Houphouët Boigny de Cocody-Abidjan pour la confirmation des noms des espèces végétales identifiées et recensées.

\section{REFERENCES}

Adeleye A, Ezekiel O, Smith S, Odusola O, Sobande J, 2008. Antibacterial activity of extracts of Alchornea cordifolia (Schum and Thonn) Mull.Arg., Boerhavia diffusa (L) and Bridellia micranthal (Hoscht) Baill. used in traditional medicine in Nigeria on Helicobacter pylori and four diarrhoeagenic bacterial pathogen. African Journal of Biotechnology Vol. 7 (20): 37613764.
Adjanohoun E \& Aké-Assi L, 1979. Contribution au recensement des plantes médicinales de Côte d'Ivoire. Centre national de la floristique, 32p.

Aké-Assi L, 1984. Flore de la Côte-d'lvoire. Étude descriptive et biogéographique avec quelques notes ethnobotaniques. Tome I. II. III. Thèse Doct. Ės-Sci. Nat., F.A.S.T. Univ. Abidjan , p. 1205.

Aké-Assi L, 2011. Abrégé de médecine et pharmacopées africaines : quelques plantes employées traditionnellement dans la 
couverture des soins de santé primaire. Edition NEI/CEDA (Côte d'Ivoire), $157 \mathrm{p}$.

Ambé ASA, Ouattara D, Tiebre M-S, Vroh BTA., Zirihi GN, N'guessan KE, 2015. Diversité des plantes médicinales utilisées dans le traitement traditionnel de la diarrhée sur les marchés d'Abidjan (Côte d'Ivoire). Journal of Animal \& Plant Sciences, Vol.26, Issue 2 , 4081-4096.

APG III, 2009. The Angiosperm Phylogeny Group, « An update of the Angiosperm Phylog-eny Group classification for the orders and families of flowering plants : APG III », Bo-tanical Journal of the Linnean Society, 161(2) : 105-121.

Awoyemi OK, Ewa EE, Abdulkarim IA, Aduloju AR, 2012. Academic research international 2: 5057

Béné K, Fofie NBY, Camara D, Kanga Y, Yapi AB, Yapo YC, Zirihi GN, 2016. Étude ethnobotanique des plantes médicinales utilisées dans le Département de Transua, District du Zanzan (Côte d'lvoire). Journal of Animal \&Plant Sciences. 27(2) : 4230-4250.

Cunningham A, 2002. Applied ethnobotany: People, wild plant use and conservation. People and Conservation Manuel, Earth scan publications Ltd, $300 \mathrm{p}$.

Diatta CD, Gueye M, Akpo LE, 2013. Les plantes médicinales utilisées contre les dermatoses dans la pharmacopée Baïnounk de Djibonker, région de Ziguinchor (Sénégal). Journal of Applied Biosciences $70: 5599$ - 5607.

El-Said F, Sofowora EA, Malcolm SA, Hofer A, 1969. An investigation into the efficacy of Ocimum gratissimum L. (Lamiaceae) as used in Nigeria native medicine. Planta Medica 17: 150-165.

Gnagne AS, Camara D, Fofié NBY, Béné K, Zirihi GN, 2017. Étude ethnobotanique des plantes médicinales utilisées dans le traitement du diabète dans le Département de Zouénoula (Côte d'lvoire), Journal of Applied Biosciences 113: 11257-11266.

Klotoé JR, Dougnon TV, Koudouvo K, Atègbo JM, Loko $F$, Akoègninou $A$, Aklikokou $K$, Dramane $K$, Gbeassor M, 2013. Ethnopharmacological survey on antihemorrhagic medicinal plants in South of Benin. European Journal of Medicinal Plants, 3(1) : 40-51

Kouakou-Siransy G, N'guessan IG, Kamenan A, Kouakou L, Gressie RB, Kablan BJ, 2012. Effet antioxydant d'Alchornea cordifolia vis $\square$ à $\square$ vis de $\mathrm{H} 2 \mathrm{O} 2$, Publication.lecames.org. Vol.(16)

Lakouéténé DPB, Ndolngar G, Berké B, Moyen J-M, Kosh Komba É, Zinga I, Silla S, MillogoRasolodimby J, Vincendeau $P$, Syssa-Magalé $\mathrm{J}$-L, Nacoulma-Ouedraogo OG, Laganier R, Badoc A, Chèze C, 2009. Enquête ethnobotanique des plantes utilisées dans le traitement du paludisme à Bangui. Bulletin De La Société de Pharmacie de Bordeaux,148: 123-138.

Mambé FT,Voukeng IK, Beng VP, 2016. Antibacterial activities of methanol extracts from Alchornea cordifolia and four other Cameroonian plants against MDR phenotypes. Journal of Taib University, Medical Sciences, 11(2): 121-127.

Mavar-Manga $\mathrm{H}$, Haddada $M$, Pieters $L$, Baccelli $C$, Penge A, Quetin-Leclercq J, 2008. Antiinflammatory compounds from leaves and root bark of Alchornea cordifolia (Schumach. \&Thonn.) Mull. Arg. Journal of Ethnopharmacology $115: 25-29$

Monnet T, 2013. Étude ethnobotanique des plantes médicinales antidiabétiques vendues sur les marchés de la commune d'Abobo, dans le District d'Abidjan (Côte d'Ivoire). Mémoire Master II de botanique, UFR Biosciences, Université Félix Houphouët-Boigny, p. 50.

Nacoulma-Ouedraogo OG, Van-Damme $P$, Zerbo $P$, 2007. Contribution à la connaissance des plantes médicinales utilisées dans les soins infantiles en pays San, au Burkina Faso. International Journal of Biological and Chemical Sciences 1(3) : 262-274.

N'guessan K, Zirihi NG, Boraud N, 2010. Étude ethnopharmacologique des plantes utilisées pour faciliter l'accouchement, en pays Abbey et Krobou, au Sud de la Côte-d'lvoire. International Journal of Biological and Chemical Sciences, 4(4) : 1004-1016.

N'guessan K, 2008. Plantes médicinales et pratiques médicales traditionnelles chez les peuples Abbey et Krobou du département d'Agboville (Côte d'lvoire). Thèse de Doctorat d'Etat ès Sciences, Université de Cocody-Abidjan, p. 235.

Ouattara D, 2006. Contribution à l'inventaire des plantes médicinales significatives utilisées dans la région de Divo (sud forestier de la Côte-d'lvoire) et à la diagnose du poivrier de Guinée : Xylopia aethiopica (Dunal) A. Rich. 
(Annonaceae). Thèse de Doctorat, Université de Cocody-Abidjan , $184 \mathrm{p}$.

Owhe-Ureghe UB, Akpo CO, 2016. In Vitro Antibacterial Activity of Alchornea cordifolia and Costusafer Against Some Bacterial Isolates. UK Journal of Pharmaceutical and Biosciences Vol. 4(3) : 51-55,

PNLP: 2005. Document de politique nationale pour la lutte contre le paludisme. Abidjan (Côte d'Ivoire): Programme National de Lutte contre le Paludisme, $18 \mathrm{p}$.

Rastogi RP, Mehrotra BN, 2002. India Central Drug Research Institute. Compendium of Indian Medicinal Plants, 3rd Vol.

Saki SJ, Mosso K, Sea TB, Diopoh KJ, 2005. Détermination de quelques composants essentiels d'amandes de Ricinodendron heudelotii en cote d'ivoire. Agronomie Africaine 17 (2) : 137-142.

Sood SK, Bhardwaj R, Lakhanpal TN, 2005. Ethnic Indian Plants in cure of diabetes. India : Scientific Publishers, Jodhpur : 77-78.

Tra Bi FH, 1997. Utilisation des plantes, par l'homme, dans les forêts classées du Haut Sassandra et de Scio, en Côte d'Ivoire, Thèse de Doctorat 3ème cycle, Fast, Université de Cocody, Abidjan, $215 p$.

Tra Bi FH, Irié GM, N'Gaman KCC, Mohou CHB, 2008. Études de quelques plantes thérapeutiques utilisées dans le traitement de l'hypertension artérielle et du diabète : deux maladies émergentes en Côte d'lvoire. Sciences et Nature 5(1) : 39-48.

Yapi AB, Kassi NJ, Fofie NBY, 2015. Etude ethnobotanique des Asteraceae médicinales vendues sur les marches du district autonome d'Abidjan (Côte d'Ivoire). International Journal of Biological and Chemical Sciences 9(6) : 2633-2647.

Zirihi GN, 1991. Contribution au recensement, à l'identification et à la connaissance de quelques espèces végétales utilisées dans la médecine traditionnelle et la pharmacopée chez les Bété du Département d'Issia, Côte d'Ivoire. Thèse de Doctorat de 3è Cycle, Université d'Abidjan , 253 p.

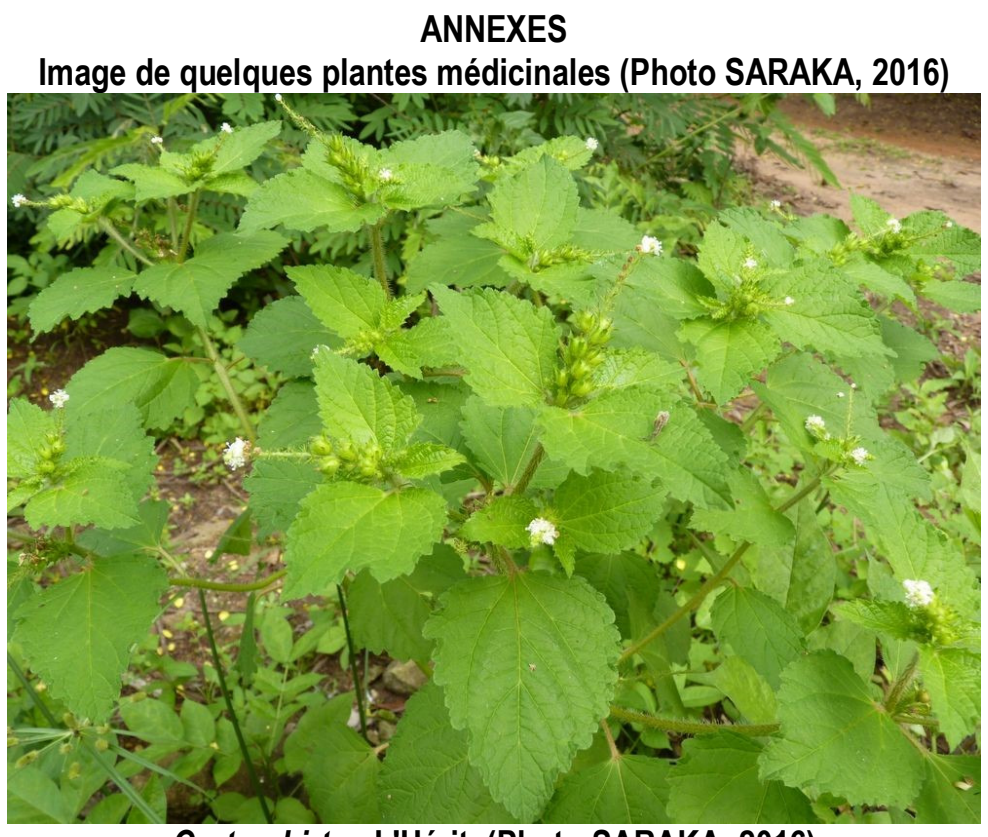

Croton hirtus L'Hérit. (Photo SARAKA, 2016)

Nom Baoulé: Ahoublé aliè 
Saraka et al, J. Appl. Biosci. 2018 Enquête ethnobotanique sur les Euphorbiaceae médicinales utilisées chez les Baoulé du District de Yamoussoukro (Côte d'Ivoire)

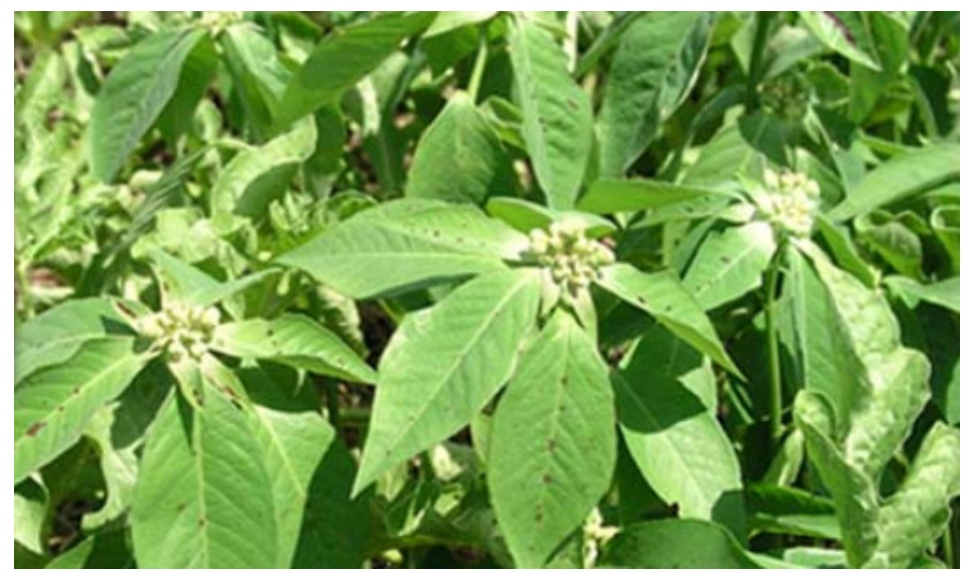

Euphorbia hétérophylla L. (Photo SARAKA, 2016)

Nom Baoulé: Gbamloaliè

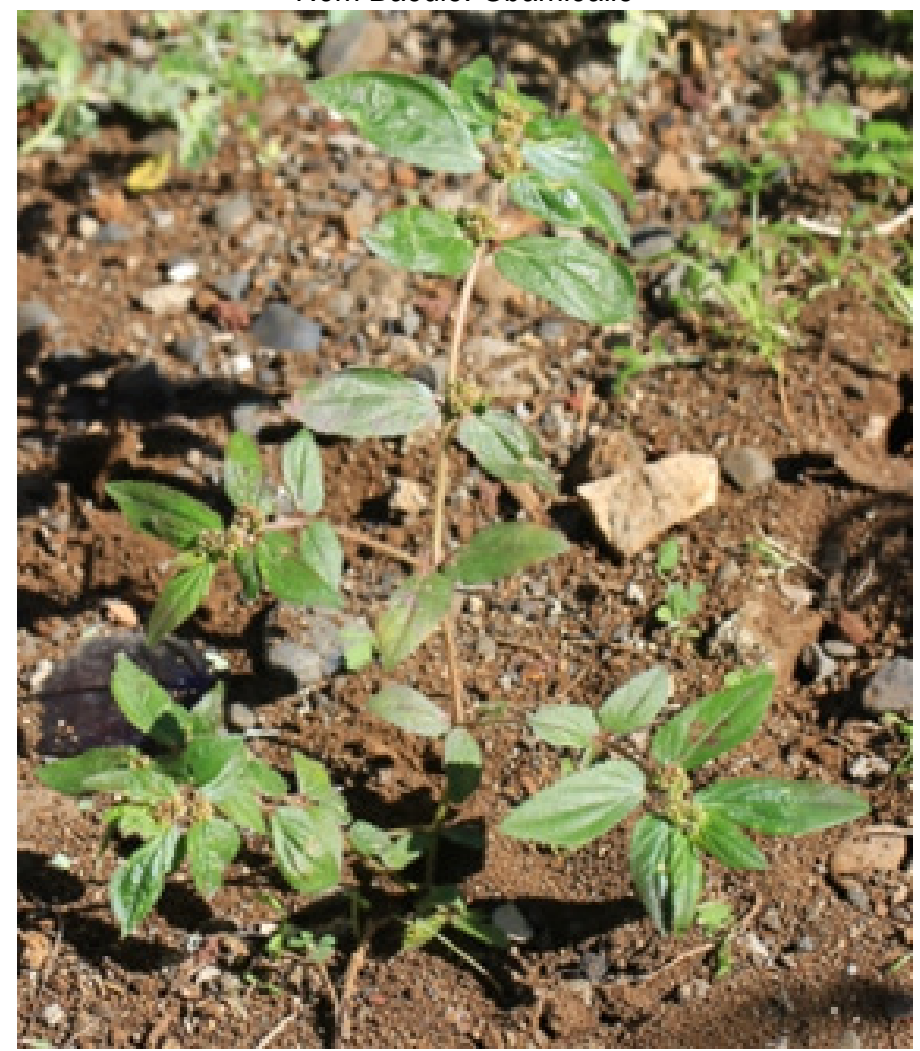

Euphorbia hirta L. (Photo SARAKA, 2016)

Nom Baoulé: Akolôlô 
Saraka et al, J. Appl. Biosci. 2018 Enquête ethnobotanique sur les Euphorbiaceae médicinales utilisées chez les Baoulé du District de Yamoussoukro (Côte d'Ivoire)

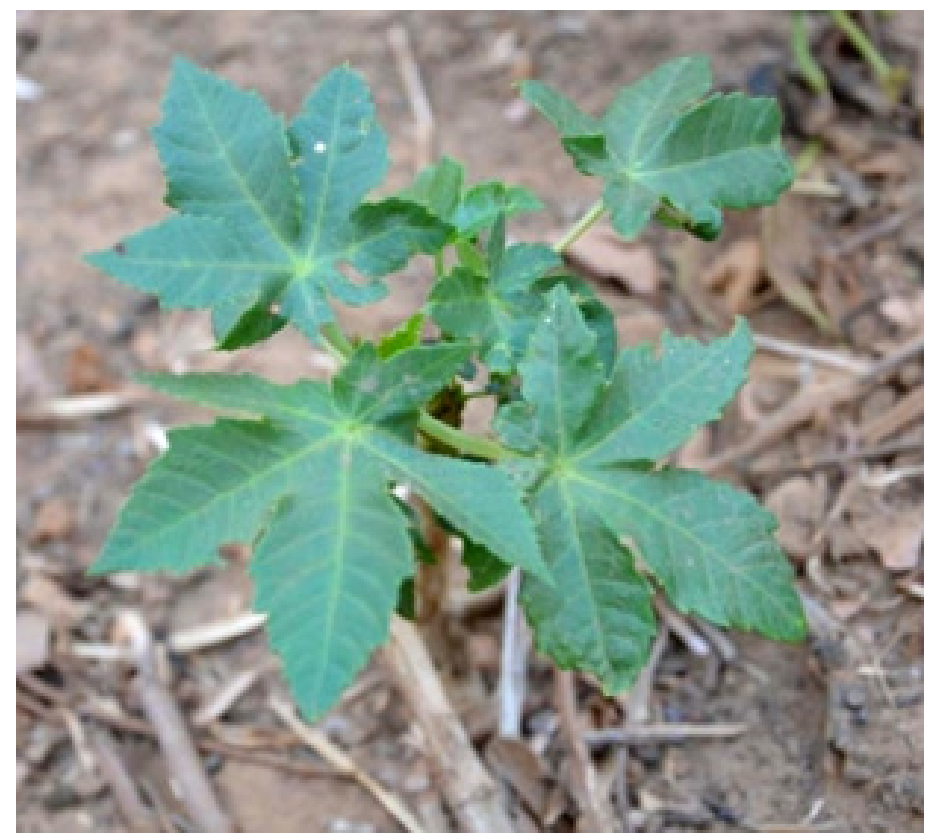

Ricinus communis L. (Photo SARAKA, 2016)

Nom Baoulé: Atténdé

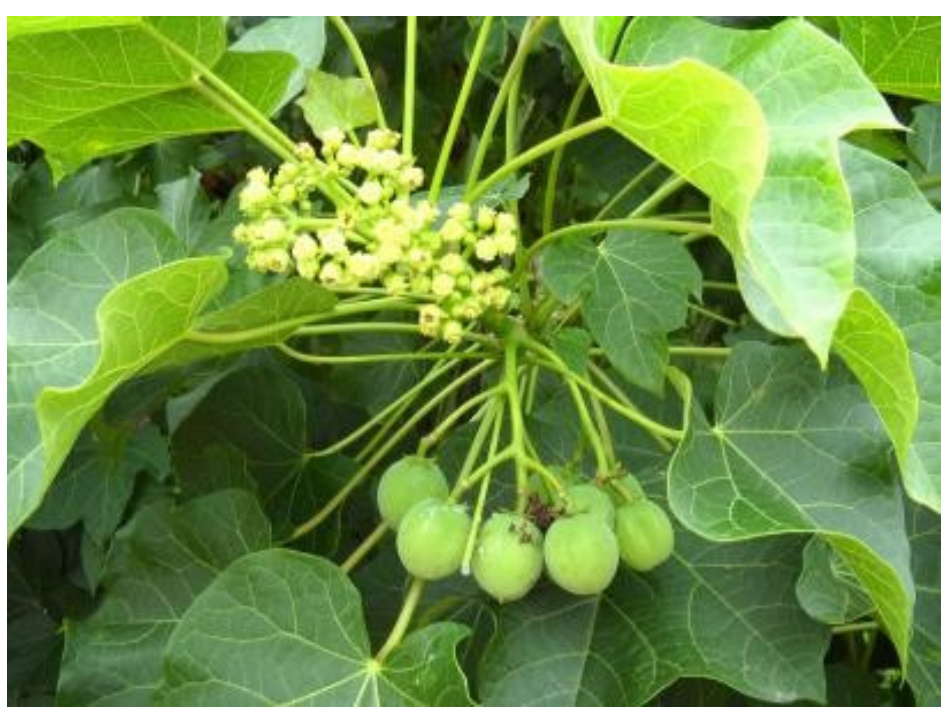

Jatropha curcas (Photo SARAKA, 2016)

Nom Baoulé: Aplôhplôh 
Saraka et al, J. Appl. Biosci. 2018 Enquête ethnobotanique sur les Euphorbiaceae médicinales utilisées chez les Baoulé du District de Yamoussoukro (Côte d'Ivoire)

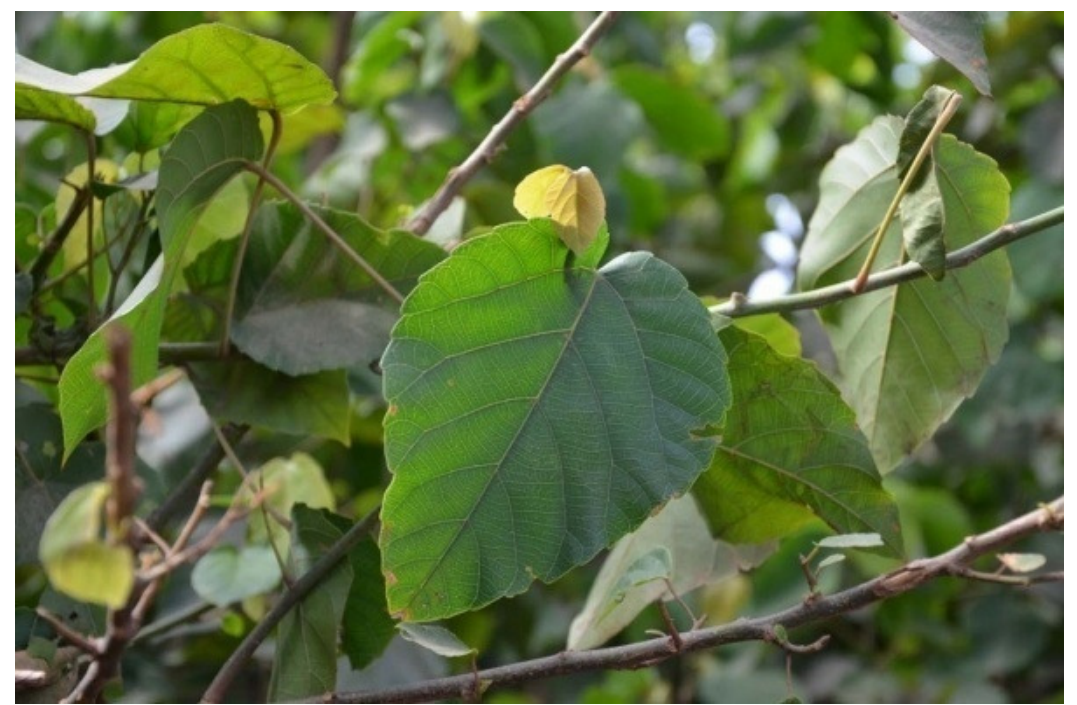

Alchornea cordifolia (Schumach\&Thonn.)Müll. Arg. (Photo SARAKA, 2016)

Nom Baoulé: Djéka 